\section{AB0728 RHEUMATIC MANIFESTATIONS OF INFLAMMATORY BOWEL DISEASES, STUDY FROM MIDDLE EAST}

M. Hammoudeh ${ }^{1}$, S. Alkaabi ${ }^{1}$, M. Sharma ${ }^{1}$, E. Al-Sayed ${ }^{1}$, P. Chandra ${ }^{2}$, M. Elbadri ${ }^{1}$, S. Hammoudeh ${ }^{3}, \mathrm{~N}$. Abu Nahya ${ }^{2} .{ }^{1}$ Medicine; ${ }^{2}$ Research center, HMC; ${ }^{3}$ Research center, Weill Cornell College-Qatar, Doha, Qatar

Background: Musculoskeletal symptoms accompanying the diagnoses of Inflammatory bowel diseases (IBD), are seen in $6-46 \%$ of cases 1 . There are very limited data about prevalence of rheumatic manifestations of IBD from the Middle East 2.

Objectives: The goal of this study is to examine the prevalence of rheumatic manifestations among patients diagnosed with IBD.

Methods: Between 1/2/2015 and 30/7/2016 all consecutive IBD patients were approached. A total of 127 adult patients signed the consent form. The diagnosis and extent of IBD (ulcerative colitis or Crohn's disease) had to be confirmed by a colonoscopy and histopathology. Patients were then interviewed and examined by one of two expert rheumatologists. A set of questions were used, complete rheumatological examination, X-rays of the lumbosacral and SI joints, and HLA-B27 test were done.

Results: Among our sample; $66 \%$ were Arabs and $34 \%$ are Asians, $58.3 \%$ were males, $52 \%$ fell in the age category of $30-49$ years, $83.1 \%$ were married, $25.6 \%$ had a graduate degree, $36.5 \%$ had a history of smoking, and $15.2 \%$ had a family history of IBD.

The sample had $36 \% \%$ with Crohn's disease, and $64 \%$ with ulcerative colitis. Any type of rheumatic manifestations were present in of $57.5 \%$ with no significant differences between the the two types of IBD diseases $(p>0.05)$. The majority of these patients had peripheral manifestations (arthralgia, arthritis, enthesitis) $(43.3 \%)$, while only $3.1 \%$ had axial alone, and $11 \%$ had both types. Among those with peripheral manifestations; $7.2 \%$ had type 1 arthritis (Pauciarticular), while $1.4 \%$ had type 2 arthritis (polyarticular). There were no significant differences between the two types of IBD diseases in regards to the presence of peripheral manifestations $(p>0.05)$. However, the two diseases were significantly different in the presence of axial manifestations as more people with Crohn's have axial manifestations (19.6\%) compared with Ulcerative colitis (12.3\%). Those with Crohn's had more people with rheumatic manifestations $4-7$ years before the diagnosis of IBD. HLA-B27 was positive in 5 patients 3 with Crohn's and 2 with ulcerative colitis. Logistic regression analysis of the data did not reveal any significant predictor or potential risk (type of IBD, gender, age group, BMI, smoking, family history, duration or extent) for the development of musculoskeletal manifestations in our patients.

Conclusions: In this study of musculosketal manifestations of patients with IBD from the Middle East $57.7 \%$ of them have any rheumatic manifestations.Peripheral manifesations occurred in $43 \%$, axial alone in $3.1 \%$, axial and peripheral manifestations in $11.5 \%$, type I arthritis in $7.2 \%$ and type II in $1.4 \%$. More patients with Crohn's has axial spondyloarthropathy (19.6\%) compared with patients with ulcerative colitis (12.3\%).

\section{References:}

[1] Atzeni F et al: Rheumatic manifestations in IBD. Autoimmunity reviews: 2014 pp 20-23.

[2] Al-Jarallah K, et al. Rheumatic complications of IBD among Arabs. Int $\mathrm{J}$ of rheum Dis 2013:16:134-138.

Disclosure of Interest: None declared

DOI: 10.1136/annrheumdis-2017-eular.1495

\section{AB0729 BENEFITS OF ADDITIONAL SPINAL MAGNETIC RESONANCE IMAGING COMPARED TO SACROILIAC JOINTS IMAGING ALONE IN THE DIAGNOSIS OF SPONDYLOARTHROPATHY}

N. Ahmad ${ }^{1}$, A. Khan ${ }^{1}$, P. Yoong ${ }^{2}$, J. Kitchen ${ }^{1}$. ${ }^{1}$ Department of Rheumatology; ${ }^{2}$ Department of Radiology, Royal Berkshire NHS Foundation Trust, Reading, United Kingdom

Background: Axial spondyloarthritis ( $\mathrm{AxSpA})$ is a chronic inflammatory condition predominantly involving the axial skeleton including the spine and sacroiliac joints. Magnetic resonance imaging (MRI) demonstrates inflammatory and structural changes in patients with both ankylosing spondylitis and non-radiographic (nrAxSpA) forms of SpA and has become widely used in diagnosing SpA.

Sacroiliitis is a prominent feature of SpA but up to $24 \%$ of patients with clinically active SpA can have normal MRI of their sacroiliac joints (SIJ). ${ }^{1}$ Spinal inflammation is well recognized in SpA and studies have shown that $49 \%$ of clinically active nr-AxSpA patients have spinal lesions alone but no lesions in SIJ. ${ }^{2}$ Thoracic spinal lesions are as common as SIJ lesions. ${ }^{3}$ Therefore using spinal in addition to SIJ MRI should improve the overall sensitivity and specificity for the detection of the disease.

Prior to publication of the 2015 EULAR recommendations, ${ }^{4}$ an evidence-based MRI protocol had been adopted in our hospital, using STIR and T1-weighted sequences of the whole spine and SIJs in patients with suspected SpA.

Objectives: To determine the additional diagnostic benefit of including limited sequence whole spine imaging with SIJ MRI to SIJ MRI alone in patients with suspected SpA.

Methods: MRI scans performed for suspected SpA over twelve months from November 2015 to November 2016 were reviewed retrospectively $(n=203)$. Reports were analysed for presence and location of lesions suggestive of SpA.
HLA-B27 status of the patients and the presence of SpA associated clinical features as defined by ASAS criteria were recorded.

Results: MRI scans from 203 patients with suspected SpA were reviewed. 81 $(40 \%)$ were male and $122(60 \%)$ were female. The age range was 13 to 78 years (mean $=41) .130(64 \%)$ were less than 45 years of age. 157/203 $(77 \%)$ patients had been tested for HLA B-27 alleles of whom 46 (29\%) were HLA-B27 positive. All patients had inflammatory back pain and $76(37 \%)$ had one or more additional SpA features as per ASAS criteria.

Overall $43(21 \%)$ patients had a positive MRI spine and/or SIJs for inflammatory or structural changes. $21 / 43(49 \%)$ patients had inflammatory lesions in their sacroiliac joints only. $18 / 43(42 \%)$ patients had inflammatory changes involving both SIJ and Spine and 4/43 (9\%) had spinal inflammatory changes only with normal SIJs (Table 1). In these four patients the thoraco-lumbar spine was involved. In HLA-B27 positive patients $(n=46), 25(54 \%)$ had a positive MRI.

\begin{tabular}{lcc}
\hline Positive Imaging Findings & Number of patients & Percentage of patients \\
\hline SIJ \& Spine & 18 & 42 \\
SIJ lesions alone & 21 & 49 \\
Spinal lesions alone & 4 & 9 \\
Total & 43 & - \\
\hline
\end{tabular}

Conclusions: The majority of patients with $\mathrm{SpA}$ can have their diagnosis confirmed on SIJ MRI. However a proportion of patients (9\%) had spinal changes only. Additional spinal MRI has been shown to increase the diagnostic yield for axial spondyloarthropathy in our cohort.

References:

[1] Bennett AN, et al Arthritis\&Rheumatism 2009;60(5):1331-41.

[2] Rudwaleit M, et al.Ann Rhem Dis 2008;67:1276-81.

[3] Van der Heijde D et al. Arthritis Rheumatol 2014;66(3):667-73.

[4] Mandl P, et al. Ann Rheum Dis 2015;0:1-13. doi:10.1136/annrheumdis-2014206971.

Disclosure of Interest: None declared

DOI: 10.1136/annrheumdis-2017-eular.4851

\section{AB0730 CELL CHOLESTEROL TRANSPORT IN SPONDYLOARTHRITIDES AND ITS RESPONSE TO ANTI-RHEUMATIC DRUGS}

N. Ronda ${ }^{1}$, I. Hokstad ${ }^{2}$, G. Deyab ${ }^{3}$, D. Greco ${ }^{1}$, S. Agewall ${ }^{4,5}$, G. Hjeltnes ${ }^{6}$, J.E. Whist ${ }^{3}$, F. Bernini ${ }^{1}$, I. Hollan ${ }^{2,7,8,9} .{ }^{1}$ Department of Food and Drug, University of Parma, Parma, Italy; ${ }^{2}$ Lillehammer Hospital for Rheumatic Diseases; ${ }^{3}$ Department of Medical Biochemistry, Innlandet Hospital Trust, Lillehammer; ${ }^{4}$ Oslo University Hospital, Ullevål; ${ }^{5}$ Institute of Clinical Sciences, University of Oslo, Olso; ${ }^{6}$ Department of Medicine; ${ }^{7}$ Innlandet Hospital Trust, Lillehammer, Norway; ${ }^{8}$ Department of Medicine, Brigham and Women's Hospital; ${ }^{9}$ Harvard Medical School, Boston, United States

Background: Spondyloarthritis is associated to accelerated atherosclerosis, possibly due to chronic inflammation and lipid metabolism disturbances. Circulating lipoprotein function may be more important than concentration. In particular, cholesterol efflux capacity (CEC) of high density lipoproteins (HDL) opposes to foam cell formation and correlates inversely with cardiovascular risk ${ }^{1}$. Instead, the capacity of low density lipoproteins (LDL) to load cells with cholesterol (CLC) favors atherosclerosis. CEC and CLC may be altered independently from lipoprotein serum levels, e.g. due to chronic autoimmune inflammation or to medical therapies ${ }^{2}$.

Objectives: Our aim was to compare CEC and CLC in patients with ankylosing spondylitis (AS) and psoriatic arthritis (PsA). We also aimed to evaluate CEC and CLC modification upon anti-rheumatic therapy and their relationship to lipoprotein levels.

Methods: Patients with AS $(n=24)$ and PsA $(n=36)$ were from the observational PSARA study. Treatment was: anti-TNF agents for AS; MTX alone or in combination with an anti-TNF agent for PsA.

Serum was drawn before, after 6 weeks and after 6 months of anti-rheumatic therapy to measure CEC with a validated cell model (radioisotopic technique to measure \% cholesterol efflux on total cell cholesterol ${ }^{3}$ ) and CLC (with a macrophage model and fluorimetric measurement of cell cholesterol ${ }^{2}$ ).

Results: At baseline serum LDL and total cholesterol were higher in PsA than in AS patients. LDL, total cholesterol and HDL increased after treatment in AS, but not in PsA.

In AS, CEC increased after 6 weeks of treatment $(4.9 \pm 0.3$ vs. $5.5 \pm 0.3,95 \% \mathrm{Cl}$ : -1.09 to $-0.03, p<0.05)$, in parallel with HDL serum levels. In PSA, CEC did not differ between any of the time points.

CLC did not change with treatment in AS nor in PsA, but was overall higher in PsA than in AS patients. Despite the LDL serum level increase in AS, after 6 months of treatment the difference between CLC in PSA and AS was the most significant (34.0 \pm 1.8 in PsA vs $27.8 \pm 1.5$ in $\mathrm{AS}, \mathrm{Cl} 95 \%$ : 3.28 to $6.67, \mathrm{p}<0.05)$. In addition, after 6 months of therapy the correlation of CLC with LDL levels, present before treatment, was lost in the AS group. In the PSA group CLC did not correlate with LDL serum levels at any time point.

Conclusions: Our novel data indicate that pro-atherogenic lipoprotein dysfunction is more marked, and less responsive to anti-rheumatic treatment, in PsA than AS patients. 